\title{
Surface chemistry of perovskite nanoplatelets exhibiting circularly polarized luminescence
}

\author{
Austin Hubley ${ }^{1,2}$, Amina Bensalah-Ledoux ${ }^{1}$, Bruno Baguenard $^{1}$, Stephan Guy ${ }^{1}$, Benjamin Abécassis $^{2, \mathbb{}}$, and Benoit \\ Mahler $^{1, \bowtie}$ \\ ${ }^{1}$ Univ Lyon, Université Claude Bernard Lyon 1, CNRS, Institut Lumière Matière, F-69622, Villeurbanne, France \\ ${ }^{2}$ Univ Lyon, ENS de Lyon, CNRS, Laboratoire de Chimie, UMR 5182, F-69342, Lyon, France
}

\begin{abstract}
Chiral halide perovskite nanocrystals have many applications in next-generation optoelectronic devices due to their interaction with circularly polarized light. Through the careful selection of chiral organic surface ligands, control over the circular dichroism (CD) and circularly polarized luminescence (CPL) of these materials can be achieved. However, while recent developments of CD-active perovskites have seen significant advances, effective CPL remains a challenge. Here, we synthesize colloidal perovskite nanoplatelets exhibiting room temperature CPL with dissymmetry factors up to $g_{\text {lum }}=4.3 \times$ $10^{-3}$ and $g_{a b s}=8.4 \times 10^{-3}$. Methylammonium lead bromide nanoplatelets are synthesized with a mixture of chiral dimethyl benzyl ammonium ligands and achiral octylammonium ligands, the precise ratio of which is shown to be critical to achieving high $g$-factors. We investigate the competitive binding of these surface ligands using ${ }^{1} \mathrm{H}$ NMR, and use an equilibrium model to demonstrate the ligand affinity. The magnitude of CPL and CD is quantitatively shown to exhibit a linear correlation, such that $g_{\text {lum }}=0.40 \times g_{a b s}$. Lastly, by screening several amines with close structures, we show that subtle differences in ligand structure have significant impact on the resulting CD signal of the nanoplatelets. Our findings provide new insights for the effective design of perovskites exhibiting CPL and can facilitate the development of high-performance devices based on circularly polarized luminescence.
\end{abstract}

ligand-induced chirality | circularly polarized luminescence | colloidal nanocrystals | chiral perovskites | surface chemistry

Correspondence:

benoit.mahler@univ-1yonl. fr

benjamin. abecassiseens-lyon.fr

\section{Introduction}

Chiral materials exhibit preferential interactions with lefthanded or right-handed circularly polarized light. The unequal absorption or emission of circularly polarized light is referred to as circular dichroism (CD) or circularly polarized luminescence (CPL), and leads to many practical technologies such as 3D displays, spintronics, quantum computing, drug screening, anti-counterfeiting, and photoelectric devices(1-6). However, realizing such technologies requires the development of sources of circularly polarized light. Conventionally this involves filtering unpolarized light using an optical polarizer, the drawbacks of which include both a decrease in intensity, and the need for additional optical elements. Current methods seek to circumvent these issues by producing direct sources of CPL, leading to device miniaturization, lower production costs, lower energy consumption, and faster data processing rates(7). Small organic chiral flu- orophores can be used for this purpose, but typically suffer from weak CPL with luminescence dissymmetry values on the order of $g_{\text {lum }}=10^{-4}$ to $g_{\text {lum }}=10^{-2}(8,9)$. Additionally, many of these organic molecules show optical activity in the UV-range, whereas applications often require emission in the visible spectrum. One of the solutions is to couple chiral organic molecules with semiconductor nanocrystals (NCs), consequently achieving direct CPL in the visible spectrum(10). Doing so, it is possible to achieve much stronger chiroptical signals $(11,12)$.

Ligand-induced chirality has been shown to induce optical activity in semiconductor nanocrystals(13). This involves the incorporation of chiral ligands either directly in the synthesis of colloidal nanocrystals, or through post-synthetic surface treatment(14). This can result in new optical activity at the characteristic wavelengths corresponding to excitonic transitions of the NC, and both circular dichroism and circularly polarized luminescence can be observed(15). However, materials showing CPL are less common than those exhibiting $\mathrm{CD}(16)$. Furthermore, to produce high-performance CPL emitters through ligand-induced chirality requires both a chiral molecule compatible with the NC surface, and a NC with superior optoelectronic properties including narrow emission line width, high photoluminescence quantum yield, and a wide range of color tunability.

Hybrid organic-inorganic perovskite nanocrystals meet these optoelectronic prerequisites, making them ideal candidates for the development of next-generation chiroptical devices(17). In addition to excellent optoelectronic properties, these materials have the advantages of affordability, flexibility, and solution processability (18-20). Furthermore, organic-inorganic perovskites are a good foundation for designing optically active NCs due to the wide variety of compatible chiral organic ligands available. Typically, these molecules consist of a chiral centre adjacent to a primary ammonium moiety which can be inserted into the perovskite framework (21). A number of reports have shown that perovskites can be prepared directly in the presence of chiral ligands, resulting in optically active nanocrystals (22-24). However, while significant progress has been made in the field of chiral perovskite materials, CPL in these materials has received few reports, particularly in the case of colloidal nanocrystals $(25,26)$. This is because the optical properties of these nanocrystals are strongly dependent on the surface chemistry, and the impact of chiral ligands remains poorly understood. Hence, this work aims to provide insight into the 
relationship between chiral organic surface ligands, and the resulting chiroptical properties in hybrid organic-inorganic perovskite nanocrystals.

Here, we report colloidal perovskite nanoplatelets exhibiting room-temperature CPL. Using a nonsolvent recrystallization process, we show that a precise ratio of chiral and achiral ligands is crucial for achieving high chiroptical responses. We develop an ${ }^{1} \mathrm{H}$ NMR method to establish a relationship between chiroptical properties and the ratio of chiral and achiral ligands, finding that an equilibrium model can be used to understand the competitive binding of chiral ligands on the NPL surface. The correlation between the absorption and luminescence dissymmetry factors is quantitatively investigated to elucidate a linear relationship between $g_{l u m}$ and $g_{a b s}$. The effects of small differences in ligand structure on the CD signal at the NC excitonic transition are compared. These results lead to a better understanding of ligand-induced chirality in perovskite nanoplatelets, and help in the strategic design of tunable sources of circularly polarized light.

\section{Results and discussion}

Methylammonium lead bromide perovskite nanoplatelets (NPLs) were prepared using a ligand-assisted re-precipitation (LARP) approach (see Methods section for details). NPLs were synthesized with a stoichiometric ratio of precursors corresponding to the thickness of a single unit cell. This is denoted as $n=2$ in terms of the number of leadbromide octahedra $n$, in the form $\mathrm{L}_{2}\left[\mathrm{MAPbBr}_{3}\right]_{n-1} \mathrm{PbBr}_{4}$, whereby L represents the organic ligands. In this work, chiral perovskites were prepared using a ligand mixture of octylammonium (OctAm) bromide and either $(R)-(+)-\alpha, 4-$ dimethylbenzylammonium ( $R$-DMBA) bromide or $(S)-(-)$ $\alpha, 4$-dimethylbenzylammonium ( $S$-DMBA) bromide. Typically, a molar fraction of $[\mathrm{DMBA}] /[\mathrm{OctAm}]=0.825$ was used unless denoted otherwise. For simplicity, these chiral NPL enantiomers are hereby denoted as $R$ - or $S$-DMBA NPLs. The achiral perovskites were prepared using only OctAm ligands.

Scanning transmission electron microscopy (STEM) images of the $R$-DMBA NPLs show well-dispersed nanoplatelets of roughly circular morphology and a mean diameter of $65 \pm 12$ $\mathrm{nm}$, with contrast homogeneity indicating a uniform thickness (Figure 1a). Additional STEM images of $R$ - and $S$ DMBA NPLs and achiral NPLs are shown in supplementary information (Figure S1). The corresponding powder X-ray diffraction (PXRD) pattern of this sample is shown in Figure 1b. No apparent differences are observed between PXRD patterns of the $S$ - and $R$-DMBA NPLs (Figure S2). Peaks resulting from periodic reflections of stacked nanoplatelets in the powder are indicated, corresponding to a stacking periodicity of $2.6 \mathrm{~nm}$. A single $\mathrm{MAPbBr}_{3}$ perovskite layer has a thickness of $1.2 \mathrm{~nm}(27)$. Thus, the remaining $1.4 \mathrm{~nm}$ can be attributed to interdigitated OctAm and DMBA ligands which have respective lengths of $1.1 \mathrm{~nm}$ and $0.8 \mathrm{~nm}$ (Figure 1c). This is consistent with values reported previously in literature for $n=2$ methylammonium lead bromide nanoplatelets and for bulk perovskites $(28,29)$.
We systematically changed the molar ratio between chiral and achiral ligands in the synthesis, and observed that the magnitude of the $\mathrm{CD}$ signal strongly depends on this ratio, in accordance with previous reports for perovskite $\operatorname{NCs}(25,30)$. We observed that the maximum $\mathrm{CD}$ signal was obtained at a molar fraction of $[\mathrm{DMBA}] /[\mathrm{OctAm}]=0.825$. This is the composition that was chosen for optical and structural characterization. The circular dichroism and circularly polarized luminescence are shown alongside the corresponding absorption and fluorescence spectra of the chiral NPLs (Figure 2a,b). The exciton absorption maximum at $430 \mathrm{~nm}$ with fluorescence emission maximum at $438 \mathrm{~nm}$ indicate a NPL thickness of $n=2(28)$. These samples show a luminescence dissymmetry $g$-factor, $g_{l u m}$ of $4.0 \times 10^{-3}$ calculated as: $g_{\text {lum }}=2 \times\left(I_{L}-I_{R}\right) /\left(I_{L}+I_{R}\right)$, where $I_{L}$ and $I_{R}$ are the intensities of the left- and right-handed components of the emitted light at the $438 \mathrm{~nm}$ emission maximum. The corresponding $g_{a b s}$ at the $430 \mathrm{~nm}$ absorption maximum is $8.4 \times 10^{-3}$, defined as $g_{a b s}=C D_{(m \text { deg })} /(32980 \times A)$, where $C D_{\text {mdeg }}$ is the circular dichroism signal in millidegrees, and $A$ is the absorbance at the given wavelength. To the best of our knowledge, these $g$-values are the highest reported for colloidal perovskite NPLs to date. Figure $2 b$ shows $g_{a b s}$ over the full spectrum, with the corresponding absorbance. The feature observed in the region near $300 \mathrm{~nm}$ can be attributed to a ligand-NC charge transfer band, with the bisignate peak in the region of 400-450 nm corresponding to the perovskite excitonic transition $(31,32)$. Spectra for the $R$ - and $S$-DMBA NPLs appear as mirror images of one another for both CD and CPL. As expected, no chiroptical features are observed in the visible spectrum for either the $R$ or $S$-DMBA-Br ligands alone, or for the achiral NPLs synthesized with purely OctAm ligands (Figures S3 and S4).

It follows that the optical properties are highly dependant on the surface chemistry of the nanoplatelets, as ligand binding alters the electronic energy levels of surface atoms(33). Therefore, to understand the role of the different ligands involved, a better understanding of the surface chemistry must be developed. Due to the structural differences between the ligands, it is expected that they would have different binding affinities for the surface of the nanoplatelets(34). Furthermore, only a small portion of the total ligands present are required to passivate the surface because the NPLs are synthesized in the presence of an excess of ligands. Therefore, the ligand composition at the surface of the nanoplatelets is not necessarily equal to the initial composition in solution. Thus, ${ }^{1} \mathrm{H}$ NMR was used to compare the relative concentration of free ligands to that of ligands bound to the nanoplatelet surface.

A detailed procedure for the relative concentration determination of ligands through ${ }^{1} \mathrm{H}$ NMR is described under Experimental Methods. In short, this involved centrifuging the platelets to remove unbound ligands before dissolving the precipitate in a polar deuterated solvent. When the NPLs are dispersed in a polar solvent, they dissolve into their constituent precursors because the initial formation of the platelets occurs as a result of a nonsolvent crystallization 

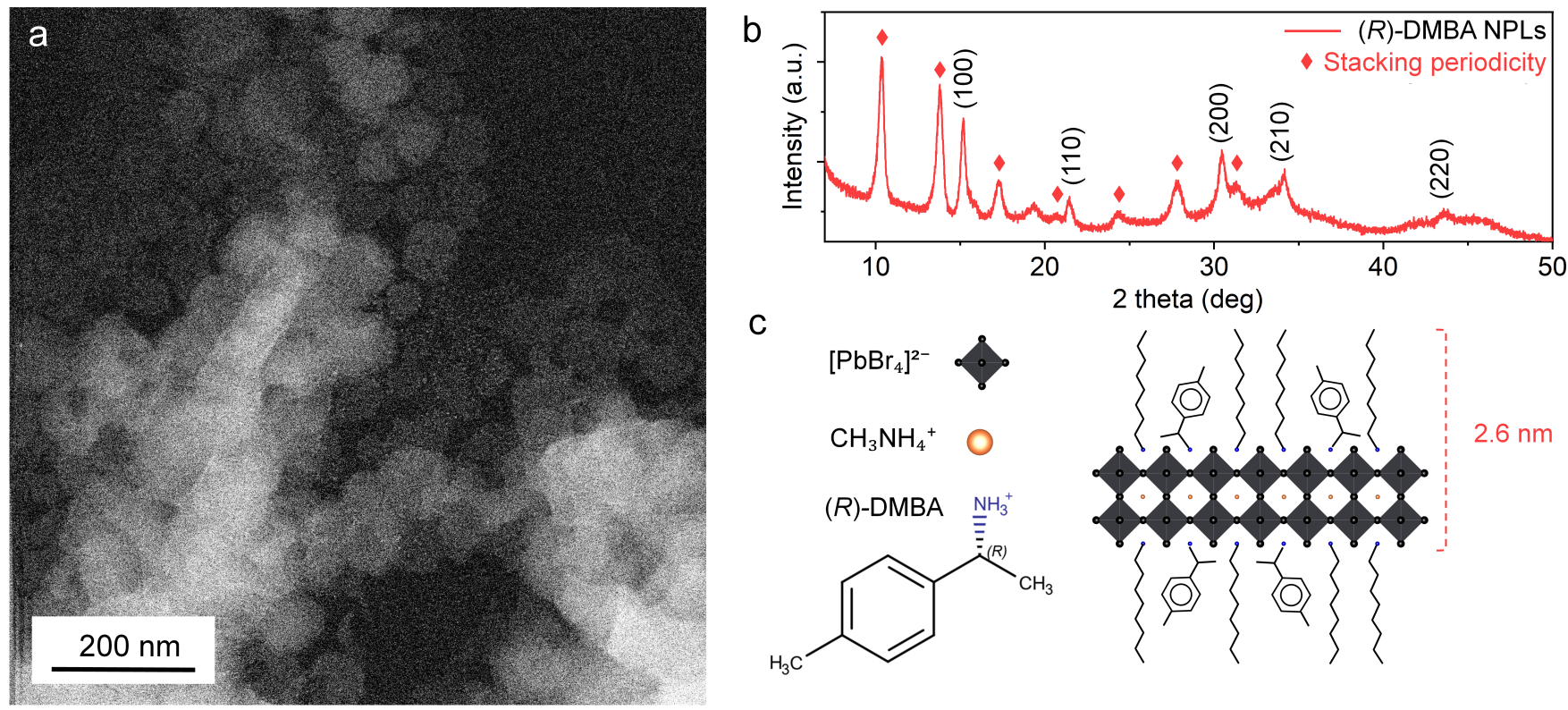

Fig. 1. (a) HAADF-STEM image of $R$-DMBA NPLs, (b) PXRD pattern of $R$-DMBA NPLs with diamonds at repeating intervals of $3.4^{\circ}$ indicating stacking periodicity of $2.6 \mathrm{~nm}$, (c) schematic illustration of corresponding NPL composition with chemical structure of $(R)-(+)$ - $\alpha$,4-dimethylbenzylammonium ligand.
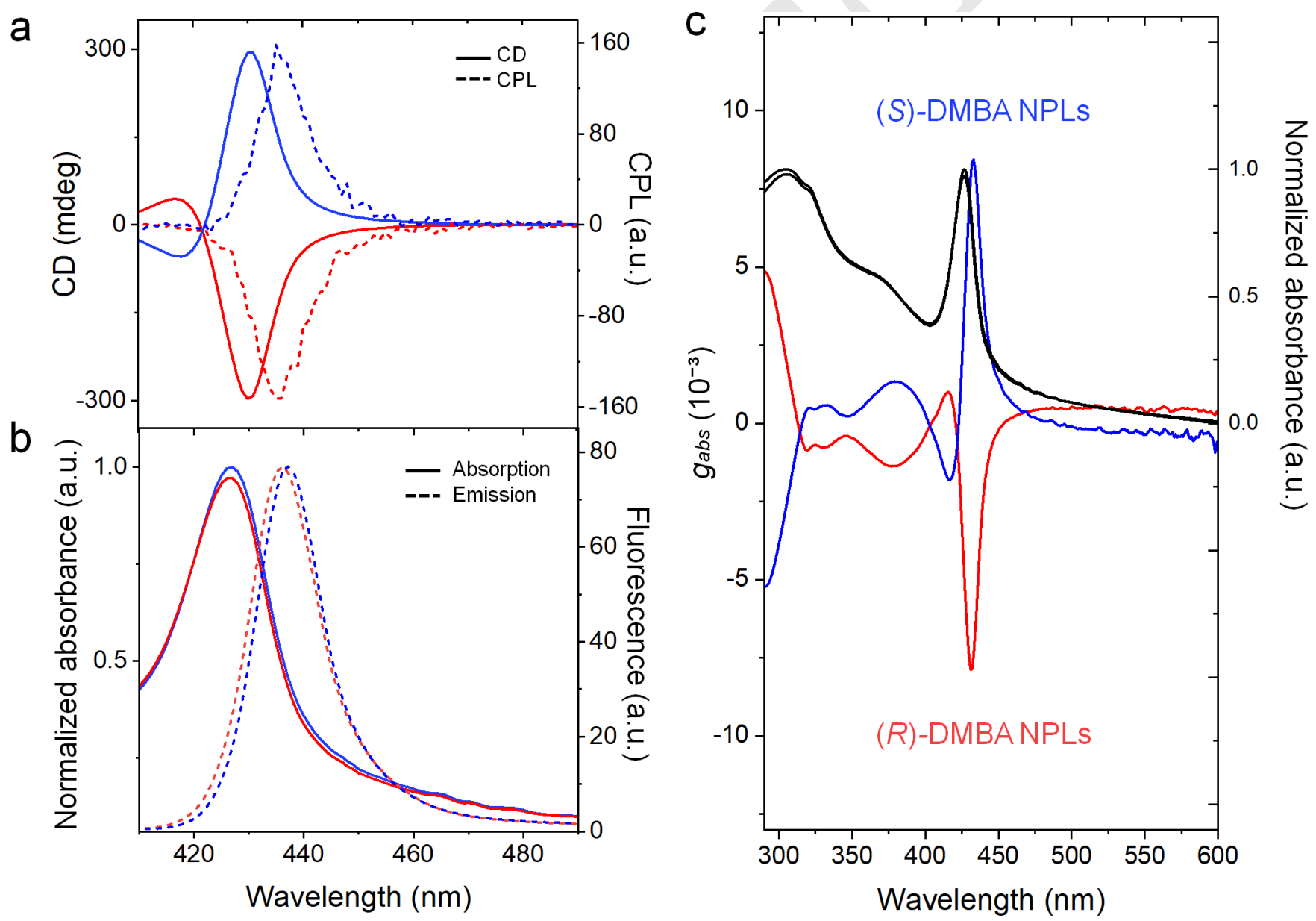

Fig. 2. (a) CD and CPL in region of NPL excitonic transition, (b) corresponding absorption and fluorescence spectra, (c) $g_{a b s}$-factors and absorbance over full spectrum. 


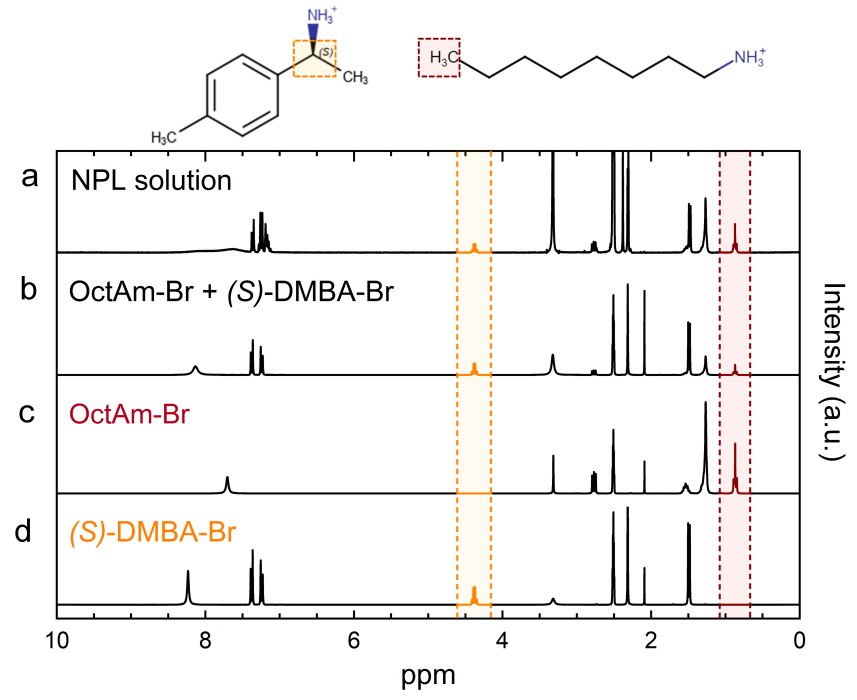

Fig. 3. ${ }^{1} \mathrm{H}$ NMR spectrum indicating signals used for relative quantification of ligands. Ligand structure with indicated protons and corresponding peaks are highlighted for DMBA (orange) and OctAm (red). Spectra are shown for (a) solution containing dissolved NPL salts including both DMBA and OctAm, (b) solution containing only DMBA and OctAm salts, (c) solution containing octylammonium bromide, (d) solutions containing only (S)-DMBA-Br.

process. This allows for sharp signals from free ligands to be resolved in the ${ }^{1} \mathrm{H}$ NMR spectrum, as there is no peak broadening resulting from bound ligands(35). Additionally, because excess ligands were removed during washing, an accurate quantification of only the ligands which were previously bound to the surface can be deduced. To do so, peaks from the ${ }^{1} \mathrm{H}$ NMR spectrum with no overlapping signals were used for relative concentration determination with the following equation:

$$
M=\left[\frac{I_{\mathrm{OctAm}}}{I_{\mathrm{DMBA}}} \times \frac{N_{\mathrm{DMBA}}}{N_{\mathrm{OctAm}}}+1\right]^{-1}
$$

Where $I$ is the integral of the signal, $N$ is the number of nuclei giving rise to the signal, and $M$ is the molar fraction of DMBA ranging between 0 and 1 . The signals used for comparison were the alpha proton of DMBA $(\delta=4.38 \mathrm{ppm})$ and the methyl protons of OctAm $(\delta=0.87 \mathrm{ppm})$, as indicated in Figure 3. This procedure was repeated for samples synthesized with different molar fractions of chiral and achiral ligands, ranging from $100 \%$ (purely chiral DMBA, $M=1$ ) down to $0 \%$ (purely OctAm, $M=0$ ). From this, an equilibrium of the ligands with the NPL surface was thus established and correlated with the magnitude of $g_{a b s}$ (Figure 4a,b).

The binding equilibrium between the different ligands and nanoplatelet surface can be expressed as follows:

$$
[\mathrm{DMBA}]+[\mathrm{NPL} \mathrm{OctAm}] \rightleftharpoons[\mathrm{OctAm}]+\left[\mathrm{NPL}_{\mathrm{DMBA}}\right]
$$

The equilibrium constant $K$ can then be written as:

$$
K=\frac{\left[\mathrm{NPL}_{\mathrm{OctAm}}\right][\mathrm{DMBA}]}{\left[\mathrm{NPL}_{\mathrm{DMBA}}\right][\mathrm{OctAm}]}=\frac{(1-M)}{M} \times \frac{x}{(1-x)}
$$

Where $x$ is the molar fraction of DMBA in solution, and again $M$ is equivalent to the molar fraction of DMBA on the surface of the nanoplatelet. Therefore, $K=1$ would be indicative of equal affinity of the ligands for the NPL surface. Fitting the experimental values using this equilibrium model results in $K=7.3 \pm 3.2$ and reveals the competitive binding between the ligands and the NPL surface, showing that OctAm has a much higher affinity than DMBA for the NPL surface, to such an extent that when $82.5 \%$ of ligands in solution consist of DMBA at the beginning of the synthesis, the percentage on the surface after the synthesis is only $33 \%$. This composition corresponds to the ratio at which the maximum CD signal is observed.

The dependence of $g_{a b s}$ on the fraction of chiral ligands at the $430 \mathrm{~nm}$ absorption maximum can be rationalized in a number of ways when considering both the ligand affinity to the nanoplatelet surface and the absorption spectra of samples synthesized with different ligand fractions (Figure 4c). As the ratio of chiral to achiral ligands is changed, the formation of different species is favoured. However, the equilibrium of ammonium ligands to the lead bromide surface is independent of the platelet morphology, and thus intercomparison of the different species is possible. In the presence of purely chiral ligands, an absorption maximum at $380 \mathrm{~nm}$ occurs, resulting from the formation of distorted lead-halide octahedra which can be attributed to a (110) perovskite with layered corrugated inorganic sheets(36). Profile plot STEM images of stacked platelets show the presence of multiple thicknesses including $n=1$, which is confirmed by PXRD indicating the majority population has a stacking periodicity of $1.6 \mathrm{~nm}$ (Figure S5). After accounting for the $0.6 \mathrm{~nm}$ lead-bromide octahedra, the remaining $1.0 \mathrm{~nm}$ can be attributed to interdigitated ligands. A CD signal on the order of $g_{a b s}=10^{-4}$ is observed in this region of $380 \mathrm{~nm}$, as well as a smaller signal at $430 \mathrm{~nm}$, resulting from the $n=1$ and $n=2$ layers, respectively (Figure S7a). This preferential formation of distorted $n=1$ species at high chiral ligand fraction is a result of the bulkyness of DMBA, because flexibility of the single lead bromide octahedra layers allows for a larger degree of distortion in the system, thus accommodating more ligands on the surface. Additionally, the absence of OctAm allows for increased $\pi-\pi$ stacking of interdigitated DMBA, further favouring the formation of a layered Ruddlesden-Popper phase structure instead of well-dispersed individual NPLs(37).

The simultaneous formation of both the $n=1$ thickness (absorption at $398 \mathrm{~nm}$ ) and the $n=2$ thickness (absorption at 430 $\mathrm{nm}$ ) can result in the low magnitude of $g_{a b s}$ due to competing CD signals from the different species. Figure S7b shows the absorption and $g_{a b s}$ spectra for NPLs synthesized with a chiral ligand fraction of 0.6 , in which both $n=1$ and $n=2$ species are present. Comparison of the CD bands between these two populations shows interfering signals. That is, the Cotton effect for $S$-DMBA NPLs in the direction of increasing wavelength is positive to negative at the $n=1$ transition, and negative to positive at the $n=2$ transition. Additionally, a correlation is seen between a decrease in the relative concentration of $n=2$ platelets and a decrease in $g_{a b s}$ when comparing the NPLs synthesized with the opposite enantiomers. The simultaneous presence of multiple species in solution will re- 

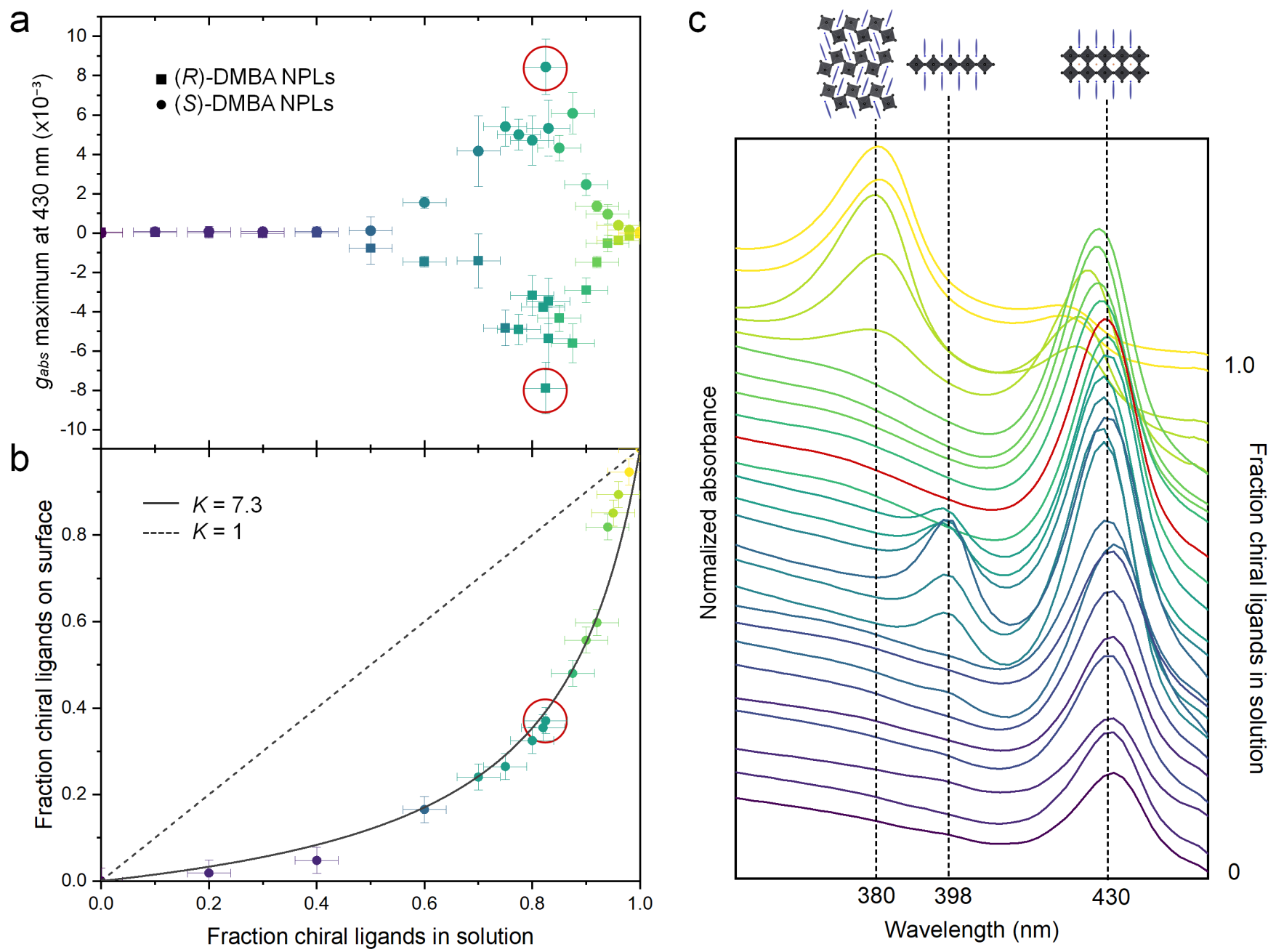

Fig. 4. (a) $g_{a b s}$ at the $430 \mathrm{~nm}$ absorption maximum for $S$ - and $R$-DMBA NPLs, (b) equilibrium curve showing competitive binding of chiral and achiral ligands to NPL surface considering $K=7.3$ (solid) and $K=1$ (dashed), (c) absorption spectra for NPLs synthesized with increasing fraction of chiral ligands in solution, with pure DMBA NPLs indicated in yellow, pure OctAm NPLs in purple, and the ligand fraction used for primary optical and structural characterization indicated in red.

sult in a CD spectrum that is the sum of all individual signals from each population. Furthermore, the CD spectrum of one species is the the sum of the differential absorption of left and right circularly polarized light. Hence, when excitonic transitions of two different species are close, the overlapping CD peaks can have cancelling effects since a positive CD signal of one population can be partially canceled by a negative signal of another thickness thus resulting in low overall signals for samples of polydisperse thickness.

The monodisperse populations of $n=2$ platelets at low molar fractions of chiral ligands (approaching 0\%) exhibit low $g_{a b s}$-factors. In this case, it simply results from an insufficient amount of chiral molecules on the surface, as the small portion of DMBA is out-competed by OctAm, which has a much higher affinity for the surface.

Of interest is the composition for which the largest $g_{a b s}$ magnitude is exhibited, corresponding to $M=0.33$. Assuming full passivation of the surface by the mixture of OctAm and DMBA, this means $1 / 3$ of surface sites are passivated by chiral ligands. At this ratio, a single species of monodisperse $n=2$ platelets are formed. The dramatic increase in CD at this specific ratio of chiral and achiral ligands can possibly result from chiral amplification due to the "sergeants-and-soldiers" effect on the nanoplatelet surface. Such a phenomenon can arise at precise ratios of chiral and achiral units when a small number of chiral units ("sergeants") control a larger portion of achiral units ("soldiers") $(12,38)$. This collective arrangement of molecules on the surface can result in long-range ordering, and chirality can be amplified beyond what is observed for ratios at which no collective interactions occur. Chiral amplification is observed when the increase of CD is non-linear in chiral molecule concentration, as can be seen in Figure S6 showing the $g_{a b s}$ maxima as a function of the fraction of chiral ligands on the nanoplatelet surface. Furthermore, the ligand concentration has influence over the geometry of the chiral molecules on the NPL surface, which can consequently affect the magnitude of the CD signal(39). This cooperative ligand effect can result in the order of magnitude increase in $g_{a b s}$ which is observed at the optimal ligand fraction. The large error associated with measurements of samples prepared near this optimal ligand fraction is a consequence of the small energetic barrier separating the formation of these different species and the different conformations of the ligands on the NC surface. 


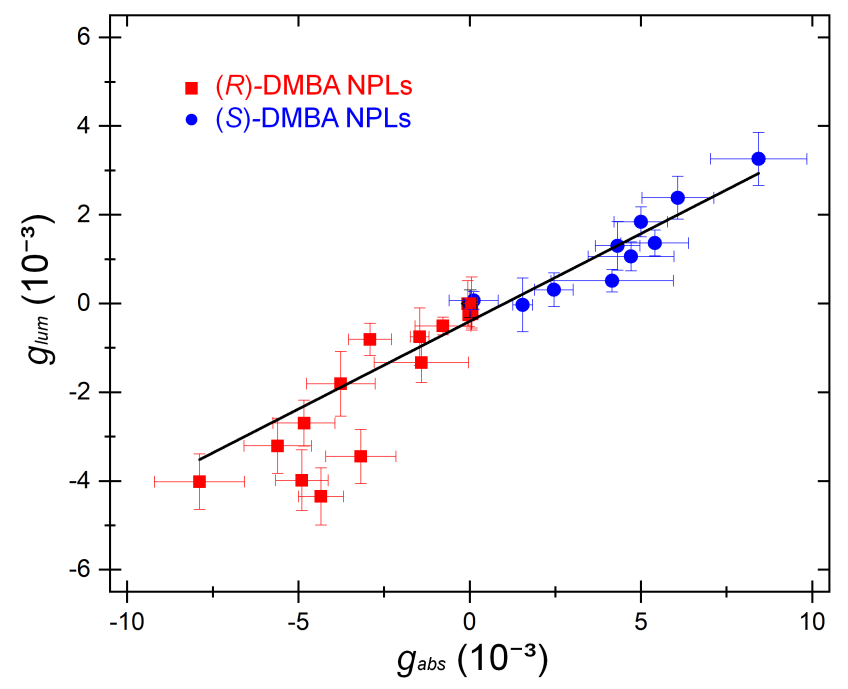

Fig. 5. Correlation of the luminescence versus absorption dissymmetry factors for NPLs prepared with different chiral ligand fractions.

Previous reports for colloidal perovskites prepared with other chiral ligands theorize that incorporation of a small amount of achiral ligands is required because the chiral molecules cannot passivate the surface alone due to their large steric profiles $(30,32)$. However, approximate calculations indicate that the packing density of chiral ligands is much greater than the density of available surface sites. The number of available surface sites for a $\mathrm{MAPbBr}_{3}$ crystal is $2.8 \mathrm{~nm}^{-2}$. The packing density of DMBA can be compared to similar aromatic chiral molecules by calculating the large-area coverage of single monolayers, arriving at an approximate density of 3.3 to 4.4 ligands $\mathrm{nm}^{-2}(29,40-42)$. Comparatively, a packing density of $\sim 4.9$ ligands $\mathrm{nm}^{-2}$ is typically exhibited for crystalline alkane chains, or a density of 4.6 $\mathrm{nm}^{-2}$ for self-assembled monolayers (SAMs) on an $\mathrm{Au}(111)$ surface $(33,35)$. From this, we see that bulky chiral ligands alone are sufficient to achieve complete passivation, suggesting that the achiral ligands have a role beyond simple surface passivation, but may contribute to long-range ordering.

To elucidate the relationship between CD and CPL, the $g_{l u m}$ value at the emission maximum was plotted against the $g_{a b s}$ value at the absorption maximum for nanoplatelets prepared with different chiral ligand fractions (Figure 5). The plot gives a regression line of $g_{l u m}=0.4 \times g_{a b s}\left(r^{2}=0.84\right)$. Negative CD and CPL signals are observed for $R$-DMBA NPLs, while the $S$-DMBA NPLs exhibit positive signals. A slight deviation to higher $g_{l u m}$ values for $R$-DMBA NPLs in comparison to the $S$-enantiomer can be attributed to the enantiomeric purity of the ligand salts, which were synthesized using precursors obtained from different suppliers. The linear correlation between the $g$-factor magnitudes is consistent with previous reports showing that $g_{l u m}$ is proportional to, and smaller than, $g_{a b s}(43)$.

Nanoplatelets exhibiting high $g_{\text {lum }}$ values will consequently also exhibit high $g_{a b s}$ values. Thus, to screen for potential NPL compositions with high dissymmetry values, the CD signals were recorded for perovskites synthesized with a number of different chiral amines. We observed a depen- dence of $g_{a b s}$ on the chiral to achiral ligand ratio for these NPLs prepared with different ligands, including $(S)$ - and $(R)$ methylbenzylamine (MBA), $(S)$ - and $(R)$-ethylbenzylamine (EBA), and $(S)$ - and $(R)$-cyclohexylethylamine (CHEA). In all cases, $n=2$ methylammonium lead bromide nanoplatelets exhibited an increased CD response when a small amount of OctAm ligands were included. The chiral ligand fraction was varied in increments of 0.02 to arrive at the optimal CD signal for each nanoplatelet, with the precise amount of OctAm required being unique to each ligand. Figure 6 compares the CD spectra of nanoplatelets synthesized with the $R$-enantiomer of each ligand. The NPL absorption spectra and corresponding CD spectra of $S$-enantiomers are shown in Figure S8. Interestingly, despite the structural similarities between each molecule and identical NC composition and thickness, significant differences are observed in the CD spectra. Simply removing the methyl group from DMBA to yield the structure of MBA results in a decrease of $g_{a b s}$ by more than an order of magnitude, but preserves the sign of the CD signal. However, altering the ligand structure between DMBA and the isomer EBA results in both a decrease in $g_{a b s}$, and an inversion in the CD signal. Replacing the aromatic ring with cyclohexane results in a $\mathrm{CD}$ band with a more pronounced Cotton effect, unlike that of the previous ligands. Instead of a single positive (or negative) band, the CD response becomes bisignate and changes sign at the wavelength where it was maximum for amines bearing an aromatic moiety. Different ligands, depending on chemical structure and composition, can split the excitonic levels of the perovskite $\mathrm{NC}$ with different efficiency, resulting in different shape and intensity of the induced CD signal(44). Even the same ligand can induce different $\mathrm{CD}$ spectra as a result of different geometrical configurations on the NC surface(14). The chirality is dependent not only on the chemistry of the ligand involved, but also on the exact geometry of binding(45). Thus, small differences in ligand structure can induce significant changes in the CD spectra, even for nanoplatelets of the same thickness and composition.

The extent to which subtle differences in ligand structure and orientation result in significant changes in CD shape and magnitude indicates that the mechanism of the optical activity observed is likely a result of chiral molecular dipole and transition dipole coupling(46). Such chirality transfer originates from coupling of the static dipole of the chiral surface ligand to the transition dipole moment of the perovskite nanocrystal, and the resulting CD spectrum is dependent on the specific binding configuration of the ligand.(46). The drastic difference in the magnitude of $g_{a b s}$ between DMBA and other chiral ligands suggests that long-range ordering may be present for this ligand, resulting in a chiral amplification which is not observed for MBA, EBA, or CHEA. Each of these ligands requires a small portion of OctAm to improve the signal, suggesting that the methods used here are a generic approach applicable to a wide range of perovskite systems. However, these results also indicate that OctAm is not required simply to help passivate the surface alongside the bulky ligands, but that inter-ligand interactions play an 


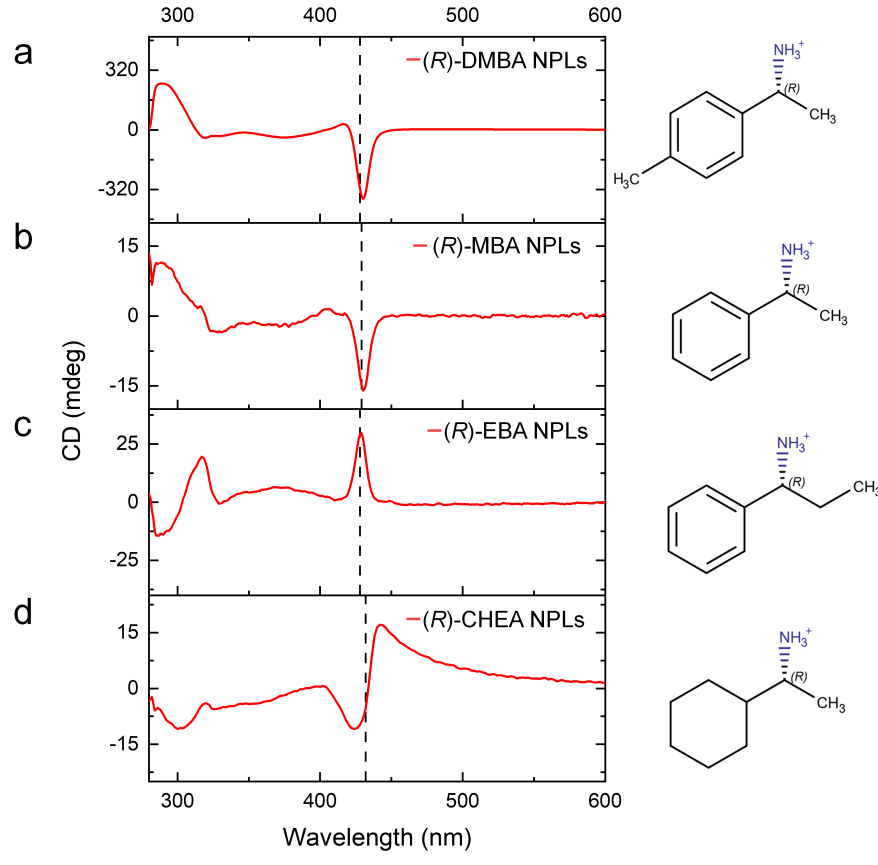

Fig. 6. $C D$ spectra of $n=2$ methylammonium lead bromide nanoplatelets synthesized with the $(R)$-enantiomer of different chiral ligands including (a) $(R)-(+)-\alpha, 4$ dimethylbenzylamine (DMBA), (b) $(R)-(+)$ - $\alpha$-methylbenzylamine (MBA), (c) $(R)$ $(+)$ - $\alpha$-ethylbenzylamine (EBA), and (d) $(R)-(-)$-1-cyclohexylethylamine (CHEA). Nanoplatelet absorption maxima are indicated by dashed lines. The chiral ligand fractions which gave the optimal responses are, respectively, $0.825,0.75,0.92$, and 0.80 , from top to bottom.

additional role in the orientation of the chiral molecules, and inhibit the formation of layered Ruddlesden-Popper phases. Furthermore, dipole-dipole coupling between the chiral ligand to the nanocrystal show that the specific choice of chiral ligand is also important to achieve optimal signals. Thus, future studies may seek to further investigate subtle structural differences between ligands to optimize chiroptical responses.

\section{Conclusions}

In conclusion, we report chiral colloidal perovskite nanoplatelets exhibiting room temperature circularly polarized luminescence. A combination of chiral and achiral molecules containing a primary ammonium moiety are incorporated as surface ligands directly in the NPL synthesis. We show that the ratio of these ligands strongly affects the chiroptical properties of the nanoplatelets, and that the competitive binding of these ligands to the nanoplatelet surface follows an equilibrium model. When the molar fraction of chiral to achiral ligands is too high or too low, formation of polydisperse thicknesses and different ligand binding orientations result in a decrease in the chiroptical signals. This approach is demonstrated to be applicable for a number of different chiral molecules. We further show that in order to optimize the magnitude of the signal, careful consideration must be given to the choice of ligand because minute structural differences result in significant changes in the magnitude and sign of the induced CD. We believe the insights provided here will help the systematic and rational development of perovskite NCs with strong chirality, leading to applications in chiroptical devices.

\section{Methods}

\section{Reagents and Materials Used.}

Lead (II) bromide $\left(\mathrm{PbBr}_{2}, 99.999 \%\right)$, methylammonium bromide (MABr, anhydrous, $\geq 99 \%$ ), n-octylammonium bromide (OctAm-Br, $\geq 99 \%$ ), hydrobromic acid ( $\mathrm{HBr}, 48$ wt\% in water), $(R)-(+)-\alpha, 4-$ Dimethylbenzylamine $(R$ DMBA, 99\%), $(S)-(-)-\alpha$-Methylbenzylamine $(S$-MBA, 98\%), $(R)-(+)-\alpha$-Methylbenzylamine $(R$-MBA, $98 \%)$, $(S)$-(+)-1-Cyclohexylethylamine ( $S$-CHEA, 98\%), N,Ndimethylformamide (DMF, anhydrous, 99.8\%), dimethyl sulfoxide- $\mathrm{d}_{6}$ (DMSO, 99.9 atom\% D), toluene (anhydrous, 99.8\%), ethanol (anhydrous), and diethyl ether (anhydrous, $\geq 99 \%$ ) were purchased from Sigma-Aldrich and used as received. Oleic acid (OA, technical grade 90\%) was purchased from Sigma-Aldrich and was dried for 1 hour at $120^{\circ} \mathrm{C}$ under vacuum before storage and use in a glovebox. $(S)-(-)$ - $\alpha, 4$-Dimethylbenzylamine $(S$-DMBA, $98 \%$ ee $99+\%),(S)-(-)-\alpha$-Ethylbenzylamine $(S$-EBA, $99+\%$, ee $99 \%), \quad(R)-(+)-\alpha$-Ethylbenzylamine ( $R$-EBA, $99+\%$, ee $98 \%)$, and $(R)-(-)-1-$ Cyclohexylethylamine ( $R$-CHEA, $98 \%$, ee $94+\%$ ) were purchased from Alfa Aesar and used as received.

Precursor preparation. Precursor salts of $S$ - and $R$-DMBA$\mathrm{Br}$ were synthesized as follows. The desired enantiomer of DMBA ( $37 \mathrm{mmol}, 5 \mathrm{~g}$ ) was dissolved in ethanol (anhydrous, $25 \mathrm{~mL}$ ) in a round bottom flask. A water bath was placed around the flask for heat dissipation and $\mathrm{HBr}(38 \mathrm{mmol}, 4.3$ $\mathrm{mL}, 48 \mathrm{wt} \%$ ) was added dropwise under vigorous stirring. After 2 hours, the flask was transferred to a rotary evaporator and dried at $70^{\circ} \mathrm{C}$ to remove volatile solvents. The resulting white crystals were transferred to a Büchner funnel and washed with cold diethyl ether ( 6 washing cycles) before being placed in an oven at $80^{\circ} \mathrm{C}$ for 1 hour. The products were transferred to an oxygen and water-free glovebox for storage. The other chiral ammonium halide salts were synthesized in a similar manner.

Nanoplatelet synthesis. All synthesis was performed in an oxygen and water-free glovebox. Stock solutions were prepared by dissolving precursor salts $\left(\mathrm{PbBr}_{2}, \mathrm{MABr}\right.$, OctAm$\mathrm{Br}, S$-DMBA-Br, $R$-DMBA-Br) in DMF to produce $0.5 \mathrm{M}$ solutions. A portion of these stock solutions were combined into a precursor solution, consisting of $20 \mu \mathrm{L} \mathrm{MABr}$, $40 \mu \mathrm{L} \mathrm{PbBr} 2,100 \mu \mathrm{L} \mathrm{OA}$, and $200 \mu \mathrm{L}$ of ammonium ligands (for instance $165 \mu \mathrm{L}$ of S-DMBA-Br and $35 \mu \mathrm{L}$ of OctAm-Br). This corresponds to a stoichiometric ratio for $\mathrm{n}=2$ nanoplatelets, with an excess of ligands for added stability. A small amount of this precursor solution $(20 \mu \mathrm{L})$ was rapidly injected into $10 \mathrm{~mL}$ of toluene under vigorous stirring, resulting in instantaneous NPL formation. For purification, platelets were centrifuged at $6000 \mathrm{rpm}$ for 5 minutes before being redispersed in $10 \mathrm{~mL}$ of toluene.

Circular Dichroism Spectroscopy. CD was measured using a Jasco J-1700 Spectrometer. Samples were measured in toluene using a $1 \mathrm{~cm}$ quartz cuvette, a 2 second integration 
time, and a $1 \mathrm{~nm}$ bandwidth. Background was subtracted with a toluene blank, and all $g_{a b s}$-factors were calculated from absorbance data measured simultaneously.

Circularly Polarized Luminescence. CPL was performed at room temperature using a home-built spectrometer described elsewhere(9). All $g_{\text {lum }}$-factors were calculated from fluorescence data measured simultaneously. Samples were excited at a 45 degree angle using a $365 \mathrm{~nm}$ diode at $20 \mathrm{~mA}$, with a $2100 \mathrm{~ms}$ integration time, a $2 \mathrm{~mm}$ slit width and $4 \mathrm{~nm}$ resolution.

Powder X-Ray Diffraction. Samples prepared under inert atmosphere were centrifuged and redispersed in a minimal amount of toluene before being drop-cast onto an air-free sample holder. PXRD measurements were performed using an Malvern Panalytical Aeris X-ray diffractometer operating at $30 \mathrm{kV}$ and $15 \mathrm{~mA}$ with a copper radiation source.

Transmission Electron Microscopy. Samples were prepared by dropcasting solutions onto an Ultrathin $\mathrm{C}$ film on lacey carbon support, 400 mesh, $\mathrm{Cu}$. (Ted Pella, Inc.) Highangle annular dark-field scanning transmission electron microscopy (HAADF-STEM) images were obtained using a Jeol microscope (JEM-ARM200F Cold FEG NeoARM) at an operating voltage of $200 \mathrm{kV}$.

${ }^{1}$ H NMR Spectroscopy. To prepare samples, the typical synthesis was scaled up to involve the injection of $120 \mu \mathrm{L}$ of precursor solution into $20 \mathrm{~mL}$ of toluene. After 5 minutes, samples were centrifuged at $6000 \mathrm{rpm}$ for 5 minutes. The supernatant was discarded, and samples were left uncovered to evaporate any residual solvent overnight. The pellet was dissolved in dimethyl sulfoxide- $\mathrm{d}_{6}(600 \mu \mathrm{L})$ and sonicated for 10 minutes before being transferred to a $5 \mathrm{~mm}$ NMR tube. Measurements were taken on a Bruker $300 \mathrm{MHz}$ spectrometer using a standard proton pulse, 16 scans, and a 2.7 second acquisition time. The signals used for relative concentration determination were the alpha proton of DMBA $(\delta=4.38$ $\mathrm{ppm})$ and the methyl protons of $\operatorname{OctAm}(\delta=0.87 \mathrm{ppm})$.

\section{ACKNOWLEDGEMENTS}

This article is part of a project that has received funding from the European Research Council (ERC) under the European Union's Horizon 2020 research and innovation programme (Grant agreement No. 865995, ERC CoG SENECA). B.M. thanks the Université de Lyon PALSE program under the framework "Investissements d'avenir" (ANR-11-IDEX-0007). We thank the Consortium Lyon St-Etienne de Microscopie (CLYM) for access to the microscope platform TEM characterization and we thank Sandrine Denis-Quanquin for access to the NMR facility.

\section{Bibliography}

1. Francesco Zinna, Umberto Giovanella, and Lorenzo Di Bari. Highly Circularly Polarized Electroluminescence from a Chiral Europium Complex. Advanced Materials, 27(10):17911795, March 2015. ISSN 09359648. doi: 10.1002/adma.201404891.

2. Jingying Wang, Chuang Zhang, Haoliang Liu, Ryan McLaughlin, Yaxin Zhai, Shai R. Vardeny, Xiaojie Liu, Stephen McGill, Dmitry Semenov, Hangwen Guo, Ryuichi Tsuchikawa, Vikram V. Deshpande, Dali Sun, and Z. Valy Vardeny. Spin-optoelectronic devices based on hybrid organic-inorganic trihalide perovskites. Nature Communications, 10(1):129, December 2019. ISSN 2041-1723. doi: 10.1038/s41467-018-07952-x.

3. H. L. Liu, Bing Zhang, Tian Gao, Xijun Wu, Fayi Cui, and Wei Xu. 3D chiral color prints for anti-counterfeiting. Nanoscale, 11(12):5506-5511, 2019. ISSN 2040-3364, 2040-3372. doi: 10.1039/C8NR09975H.

4. Ying Yang. Circularly polarized light detection by a chiral organic semiconductor transistor. Nature Photonics, 7:6, 2013

5. Finn Purcell Milton, Joseph Govan, Maria V. Mukhina, and Yurii K. Gun'ko. The chiral nanoworld: chiroptically active quantum nanostructures. Nanoscale Horizons, 1(1):14-26, 2016. ISSN 2055-6756, 2055-6764. doi: 10.1039/C5NH00072F.

6. Chao Chen, Liang Gao, Wanru Gao, Cong Ge, Xinyuan Du, Zha Li, Ying Yang, Guangda $\mathrm{Niu}$, and Jiang Tang. Circularly polarized light detection using chiral hybrid perovskite.
Nature Communications, 10(1):1927, December 2019. ISSN 2041-1723. doi: 10.1038/ s41467-019-09942-z.

7. Xiaobo Wang, Yue Wang, Weiyin Gao, Lin Song, Chenxin Ran, Yonghua Chen, and Wei Huang. Polarization-Sensitive Halide Perovskites for Polarized Luminescence and Detection: Recent Advances and Perspectives. Advanced Materials, 33(12):2003615, March 2021. ISSN 0935-9648, 1521-4095. doi: 10.1002/adma.202003615.

8. Esther M. Sanchez-Carnerero, Antonia R. Agarrabeitia, Florencio Moreno, Beatriz L. Maroto, Gilles Muller, María J. Ortiz, and Santiago de la Moya. Circularly Polarized Luminescence from Simple Organic Molecules. Chemistry - A European Journal, 21(39): 13488-13500, September 2015. ISSN 09476539. doi: 10.1002/chem.201501178.

9. Laure Guy, Maëlle Mosser, Delphine Pitrat, Jean-Christophe Mulatier, Mercedes Kukułka, Monika Srebro-Hooper, Erwann Jeanneau, Amina Bensalah-Ledoux, Bruno Baguenard, and Stéphan Guy. Modulation of Chiroptical Properties in a Series of Helicene-like Compounds. The Journal of Organic Chemistry, 84(17):10870-10876, September 2019. ISSN 0022-3263, 1520-6904. doi: 10.1021/acs.joc.9b01465.

10. Urice Tohgha, Kirandeep K. Deol, Ashlin G. Porter, Samuel G. Bartko, Jung Kyu Choi, Brian M. Leonard, Krisztina Varga, Jan Kubelka, Gilles Muller, and Milan Balaz. Ligand Induced Circular Dichroism and Circularly Polarized Luminescence in CdSe Quantum Dots. ACS Nano, 7(12):11094-11102, December 2013. ISSN 1936-0851, 1936-086X. doi: 10. $1021 / \mathrm{nn} 404832 \mathrm{f}$.

11. Assaf Ben-Moshe, Ben M. Maoz, Alexander O. Govorov, and Gil Markovich. Chirality and chiroptical effects in inorganic nanocrystal systems with plasmon and exciton resonances. Chemical Society Reviews, 42(16):7028, 2013. ISSN 0306-0012, 1460-4744. doi: 10.1039/ c3cs60139k

12. Sang Won Im, Hyo-Yong Ahn, Ryeong Myeong Kim, Nam Heon Cho, Hyeohn Kim, YaeChan Lim, Hye-Eun Lee, and Ki Tae Nam. Chiral Surface and Geometry of Metal Nanocrystals. Advanced Materials, 32(41):1905758, October 2020. ISSN 0935-9648, 1521-4095. doi: 10.1002/adma.201905758.

13. Mícheál P. Moloney, Yurii K. Gun'ko, and John M. Kelly. Chiral highly luminescent CdS quantum dots. Chemical Communications, (38):3900, 2007. ISSN 1359-7345, 1364-548X. doi: 10.1039/b704636g

14. Vera Kuznetsova, Yulia Gromova, Marina Martinez-Carmona, Finn Purcell-Milton, Elena Ushakova, Sergei Cherevkov, Vladimir Maslov, and Yurii K. Gun'ko. Ligand-induced chirality and optical activity in semiconductor nanocrystals: theory and applications. Nanophotonics, 10(2):797-824, December 2020. ISSN 2192-8614, 2192-8606. doi: 10.1515/ nanoph-2020-0473.

15. Xiaoqing Gao, Bing Han, Xuekang Yang, and Zhiyong Tang. Perspective of Chiral Colloidal Semiconductor Nanocrystals: Opportunity and Challenge. Journal of the American Chemical Society, 141(35):13700-13707, September 2019. ISSN 0002-7863, 1520-5126. doi: 10.1021/jacs.9b05973.

16. Sunihl Ma, Jihoon Ahn, and Jooho Moon. Chiral Perovskites for Next-Generation Photonics: From Chirality Transfer to Chiroptical Activity. Advanced Materials, page 2005760, April 2021. ISSN 0935-9648, 1521-4095. doi: 10.1002/adma.202005760.

17. Yangyang Dang, Xiaolong Liu, Bingqiang Cao, and Xutang Tao. Chiral halide perovskite crystals for optoelectronic applications. Matter, 4(3):794-820, March 2021. ISSN 25902385. doi: $10.1016 /$ j.matt.2020.12.018

18. Amrita Dey, Junzhi Ye, Apurba De, Elke Debroye, Seung Kyun Ha, Eva Bladt, Anuraj S. Kshirsagar, Ziyu Wang, Jun Yin, Yue Wang, Li Na Quan, Fei Yan, Mengyu Gao, Xiaoming Li, Javad Shamsi, Tushar Debnath, Muhan Cao, Manuel A Scheel, Sudhir Kumar, Julian A. Steele, Marina Gerhard, Lata Chouhan, Ke Xu, Xian-gang Wu, Yanxiu Li, Yangning Zhang, Anirban Dutta, Chuang Han, Ilka Vincon, Andrey L. Rogach, Angshuman Nag, Anunay Samanta, Brian A. Korgel, Chih-Jen Shih, Daniel R. Gamelin, Dong Hee Son, Haibo Zeng, Haizheng Zhong, Handong Sun, Hilmi Volkan Demir, Ivan G. Scheblykin, Iván Mora-Seró, Jacek K. Stolarczyk, Jin Z. Zhang, Jochen Feldmann, Johan Hofkens, Joseph M. Luther, Julia Pérez-Prieto, Liang Li, Liberato Manna, Maryna I. Bodnarchuk, Maksym V. Kovalenko, Maarten B. J. Roeffaers, Narayan Pradhan, Omar F. Mohammed, Osman M. Bakr, Peidong Yang, Peter Müller-Buschbaum, Prashant V. Kamat, Qiaoliang Bao, Qiao Zhang, Roman Krahne, Raquel E. Galian, Samuel D. Stranks, Sara Bals, Vasudevanpillai Biju, William A. Tisdale, Yong Yan, Robert L. Z. Hoye, and Lakshminarayana Polavarapu. State of the Art and Prospects for Halide Perovskite Nanocrystals. ACS Nano, 15(7):10775-10981, July 2021. ISSN 1936-0851, 1936-086X. doi: 10.1021/acsnano.0c08903.

19. Joseph S. Manser, Jeffrey A. Christians, and Prashant V. Kamat. Intriguing Optoelectronic Properties of Metal Halide Perovskites. Chemical Reviews, 116(21):12956-13008, November 2016. ISSN 0009-2665, 1520-6890. doi: 10.1021/acs.chemrev.6b00136.

20. Javad Shamsi, Alexander S. Urban, Muhammad Imran, Luca De Trizio, and Liberato Manna. Metal Halide Perovskite Nanocrystals: Synthesis, Post-Synthesis Modifications, and Their Optical Properties. Chemical Reviews, 119(5):3296-3348, March 2019. ISSN 0009-2665, 1520-6890. doi: 10.1021/acs.chemrev.8b00644.

21. Guankui Long, Randy Sabatini, Makhsud I. Saidaminov, Girish Lakhwani, Abdullah Rasmita, Xiaogang Liu, Edward H. Sargent, and Weibo Gao. Chiral-perovskite optoelectronics. Nature Reviews Materials, 5(6):423-439, June 2020. ISSN 2058-8437. doi: 10.1038/s41578-020-0181-5.

22. Jihoon Ahn, Eunsong Lee, Jeiwan Tan, Wooseok Yang, Bokyung Kim, and Jooho Moon. A new class of chiral semiconductors: chiral-organic-molecule-incorporating organic-inorganic hybrid perovskites. Materials Horizons, page 7, 2017.

23. Chunqing Yuan, Xinyue Li, Sergey Semin, Yaqing Feng, Theo Rasing, and Jialiang Xu. Chiral Lead Halide Perovskite Nanowires for Second-Order Nonlinear Optics. Nano Letters, 18(9):5411-5417, September 2018. ISSN 1530-6984, 1530-6992. doi: 10.1021/acs.nanolett. $8 \mathrm{~b} 01616$.

24. Jiaqi Ma, Haizhen Wang, and Dehui Li. Recent Progress of Chiral Perovskites: Materials, Synthesis, and Properties. Advanced Materials, 33(26):2008785, July 2021. ISSN 09359648, 1521-4095. doi: 10.1002/adma.202008785.

25. Young-Hoon Kim, Yaxin Zhai, E. Ashley Gaulding, Severin N. Habisreutinger, Taylor Moot, Bryan A. Rosales, Haipeng Lu, Abhijit Hazarika, Roman Brunecky, Lance M. Wheeler, Joseph J. Berry, Matthew C. Beard, and Joseph M. Luther. Strategies to Achieve High Circularly Polarized Luminescence from Colloidal Organic-Inorganic Hybrid Perovskite 
Nanocrystals. ACS Nano, 14(7):8816-8825, July 2020. ISSN 1936-0851, 1936-086X. doi: 10.1021/acsnano.0c03418.

26. Wenjie Chen, Shuai Zhang, Minghao Zhou, Tonghan Zhao, Xujin Qin, Xinfeng Liu, Minghua Liu, and Pengfei Duan. Two-Photon Absorption-Based Upconverted Circularly Polarized Luminescence Generated in Chiral Perovskite Nanocrystals. The Journal of Physical Chemistry Letters, 10(12):3290-3295, June 2019. ISSN 1948-7185, 1948-7185. doi: 10.1021/acs.jpclett.9b01224.

27. Pooja Tyagi, Sarah M. Arveson, and William A. Tisdale. Colloidal Organohalide Perovskite Nanoplatelets Exhibiting Quantum Confinement. The Journal of Physical Chemistry Letters, 6(10):1911-1916, May 2015. ISSN 1948-7185. doi: 10.1021/acs.jpclett.5b00664.

28. Mark C. Weidman, Michael Seitz, Samuel D. Stranks, and William A. Tisdale. Highly Tunable Colloidal Perovskite Nanoplatelets through Variable Cation, Metal, and Halide Composition. ACS Nano, 10(8):7830-7839, August 2016. ISSN 1936-0851, 1936-086X. doi: 10.1021/ acsnano.6b03496.

29. Kai-Hung Wang, Liang-Chen Li, Muthaiah Shellaiah, and Kien Wen Sun. Structural and Photophysical Properties of Methylammonium Lead Tribromide (MAPbBr3) Single Crystals. Scientific Reports, 7(1):13643, December 2017. ISSN 2045-2322. doi: 10.1038/ s41598-017-13571-1.

30. He Ren, Yue Wu, Chenchen Wang, and Yong Yan. 2D Perovskite Nanosheets with Intrinsic Chirality. The Journal of Physical Chemistry Letters, 12(10):2676-2681, March 2021. ISSN 1948-7185, 1948-7185. doi: 10.1021/acs.jpclett.1c00315.

31. Brian P. Bloom, Vankayala Kiran, Vaibhav Varade, Ron Naaman, and David. H. Waldeck. Spin Selective Charge Transport through Cysteine Capped CdSe Quantum Dots. Nano Letters, 16(7):4583-4589, July 2016. ISSN 1530-6984, 1530-6992. doi: 10.1021/acs.nanolett. $6 \mathrm{~b} 01880$.

32. Zheni N. Georgieva, Brian P. Bloom, Supriya Ghosh, and David H. Waldeck. Imprinting Chirality onto the Electronic States of Colloidal Perovskite Nanoplatelets. Advanced Materials, 30(23):1800097, June 2018. ISSN 0935-9648, 1521-4095. doi: 10.1002/adma.201800097.

33. Sung Jun Lim, Liang Ma, André Schleife, and Andrew M. Smith. Quantum dot surface engineering: Toward inert fluorophores with compact size and bright, stable emission. Coordination Chemistry Reviews, 320-321:216-237, August 2016. ISSN 00108545. doi: 10.1016/j.ccr.2016.03.012.

34. Nicholas C. Anderson, Mark P. Hendricks, Joshua J. Choi, and Jonathan S. Owen. Ligand Exchange and the Stoichiometry of Metal Chalcogenide Nanocrystals: Spectroscopic Observation of Facile Metal-Carboxylate Displacement and Binding. Journal of the American Chemical Society, 135(49):18536-18548, December 2013. ISSN 0002-7863, 1520-5126. doi: $10.1021 / \mathrm{ja} 4086758$.

35. Nicholas C. Anderson, Peter. E. Chen, Aya K. Buckley, Jonathan De Roo, and Jonathan S. Owen. Stereoelectronic Effects on the Binding of Neutral Lewis Bases to CdSe Nanocrystals. Journal of the American Chemical Society, 140(23):7199-7205, June 2018. ISSN 0002-7863, 1520-5126. doi: 10.1021/jacs.8b02927.

36. Matthew D. Smith and Hemamala I. Karunadasa. White-Light Emission from Layered Halide Perovskites. Accounts of Chemical Research, 51(3):619-627, March 2018. ISSN 00014842, 1520-4898. doi: 10.1021/acs.accounts.7b00433.

37. Xupeng Gao, Xiangtong Zhang, Wenxu Yin, Hua Wang, Yue Hu, Qingbo Zhang, Zhifeng Shi, Vicki L. Colvin, William W. Yu, and Yu Zhang. Ruddlesden-Popper Perovskites: Synthesis and Optical Properties for Optoelectronic Applications. Advanced Science, 6(22): 1900941, November 2019. ISSN 2198-3844, 2198-3844. doi: 10.1002/advs.201900941.

38. Mark M. Green, Michael P. Reidy, Robert D. Johnson, Graham Darling, Daniel J. O'Leary, and Grant Willson. Macromolecular stereochemistry: the out-of-proportion influence of optically active comonomers on the conformational characteristics of polyisocyanates. The sergeants and soldiers experiment. Journal of the American Chemical Society, 111(16): 6452-6454, August 1989. ISSN 0002-7863, 1520-5126. doi: 10.1021/ja00198a084.

39. Vera A. Kuznetsova, Eric Mates-Torres, Nadezda Prochukhan, Madeline Marcastel, Finn Purcell-Milton, John O'Brien, Anastasia K. Visheratina, Marina Martinez-Carmona, Yulia Gromova, Max Garcia-Melchor, and Yurii K. Gun'ko. Effect of Chiral Ligand Concentration and Binding Mode on Chiroptical Activity of CdSe/CdS Quantum Dots. ACS Nano, 13 (11):13560-13572, November 2019. ISSN 1936-0851, 1936-086X. doi: 10.1021/acsnano. $9 \mathrm{~b} 07513$.

40. H. Lopez de Diego. (R,S)-1-Phenylethylammonium (R,S)-Mandelate. Acta Crystallographica Section C Crystal Structure Communications, 51(5):935-937, May 1995. ISSN 01082701. doi: 10.1107/S010827019401303X.

41. David E. Turkington, Elizabeth J. MacLean, Alan J. Lough, George Ferguson, and Christopher Glidewell. Supramolecular structures of 1-phenylethylammonium tartrates. Acta Crystallographica Section B Structural Science, 61(1):103-114, February 2005. ISSN 01087681. doi: $10.1107 / \mathrm{S} 0108768104029684$.

42. Melanie Rademeyer. 2-Phenylethylammonium bromide. Acta Crystallographica Section E Structure Reports Online, 63(1):0221-0223, January 2007. ISSN 1600-5368. doi: $10.1107 /$ S1600536806051488.

43. Hiroki Tanaka, Yoshihisa Inoue, and Tadashi Mori. Circularly Polarized Luminescence and Circular Dichroisms in Small Organic Molecules: Correlation between Excitation and Emission Dissymmetry Factors. ChemPhotoChem, 2(5):386-402, May 2018. ISSN 23670932. doi: 10.1002/cptc.201800015.

44. Mayank Puri and Vivian E. Ferry. Circular Dichroism of CdSe Nanocrystals Bound by Chira Carboxylic Acids. ACS Nano, 11(12):12240-12246, December 2017. ISSN 1936-0851, 1936-086X. doi: 10.1021/acsnano.7b05690.

45. Guangmin Li, Xuening Fei, Hongfei Liu, Jing Gao, Jiayang Nie, Yuanbo Wang, Zhaodong Tian, Caicai He, Jiang-Long Wang, Chao Ji, Dan Oron, and Gaoling Yang. Fluorescence and Optical Activity of Chiral CdTe Quantum Dots in Their Interaction with Amino Acids. ACS Nano, 14(4):4196-4205, April 2020. ISSN 1936-0851, 1936-086X. doi: 10.1021/acsnano. 9b09101.

46. Aaron Forde, Dibyajyoti Ghosh, Dmitri Kilin, Amanda C. Evans, Sergei Tretiak, and Amanda J. Neukirch. Induced Chirality in Halide Perovskite Clusters through Surface Chemistry. The Journal of Physical Chemistry Letters, pages 686-693, January 2022. ISSN 1948-7185, 1948-7185. doi: 10.1021/acs.jpclett.1c04060. 